\title{
Bifurcation structure and noise-assisted transitions in the Pleistocene glacial cycles
}

\author{
Peter D. Ditlevsen ${ }^{1}$ \\ Received 6 August 2008; revised 3 March 2009; accepted 15 April 2009; published 8 August 2009.
}

[1] The glacial cycles are attributed to the climatic response of the orbital changes in the irradiance to the Earth. These changes in the forcing are too small to explain the observed climate variations as simple linear responses. Nonlinear amplifications of the orbital forcing are necessary to account for the glacial cycles. Here an empirical model of the nonlinear response is presented. From the model it is possible to assess the role of stochastic noise in comparison to the deterministic orbital forcing of the ice ages. The model is based on the bifurcation structure derived from the climate history. It indicates the dynamical origin of the mid-Pleistocene transition from the "41 ka world" to the " 100 ka world." The dominant forcing in the latter is still the 41 ka obliquity cycle, but the bifurcation structure of the climate system is changed. The model suggests that transitions between glacial and interglacial climate are assisted by internal stochastic noise in the period prior to the last five glacial cycles, while the last five cycles are deterministic responses to the orbital forcing.

Citation: Ditlevsen, P. D. (2009), Bifurcation structure and noise-assisted transitions in the Pleistocene glacial cycles, Paleoceanography, 24, PA3204, doi:10.1029/2008PA001673.

\section{Introduction}

[2] The dominant orbital periods in solar insolation is the $41 \mathrm{ka}$ obliquity cycle (tilt of rotational axis, determining the meridional gradient in insolation) and the precessional cycles (determining the season when Earth is closest to the Sun) which decompose into $19 \mathrm{ka}$ and $23 \mathrm{ka}$ periods. However, through the last $800 \mathrm{ka}$ to $1 \mathrm{Ma}$ the dominant period for the glacial cycles is approximately $100 \mathrm{ka}$ similar to the 1 order of magnitude weaker eccentricity cycle (determining the semiannual difference in distance to the sun). The weakness of this climatic forcing is referred to as the $100 \mathrm{ka}$ problem of the Milankovitch theory [Imbrie et $a l ., 1993]$. It is now generally accepted that the $100 \mathrm{ka}$ glacial timescale cannot be attributed to the eccentricity cycle [Huybers, 2007]. In the Pliocene and early Pleistocene, 3-1 Ma B.P., the dominant period of variation was indeed the $41 \mathrm{ka}$ obliquity variation [Raymo et al., 1989; Huybers and Wunsch, 2005].

[3] Different mechanisms have been proposed to explain the occurrence of the $100 \mathrm{ka}$ glacial cycle. These range from self-sustained nonlinear oscillators [Källen et al., 1979; Saltzman and Sutera, 1987; Gildor and Tziperman, 2000], forced nonlinear oscillators [LeTreut and Ghil, 1983] to stochastic or coherence resonance [Benzi et al., 1982; Pelletier, 2003]. Others emphasize, on the basis of spectral analysis, the nonlinear nature [Rial, 1999] and the stochastic nature of the climate signal [Kominz and Pisias, 1979; Ashkenazy et al., 2005].

[4] The nonlinearity of the response to the forcing implies that a linear cross-spectral analysis between the paleocli-

\footnotetext{
${ }^{1}$ Centre for Ice and Climate, Niels Bohr Institute, University of Copenhagen, Copenhagen, Denmark.

Copyright 2009 by the American Geophysical Union. 0883-8305/09/2008PA001673\$12.00
}

mate record and the components of the orbital forcing does not necessarily pick out accurately the relative weights of the different components.

[5] Combined evidence from records of glaciations on land and deep sea records suggest that the climate has shifted between different quasi-stable states characterized by the mode of the global ocean circulation and the degree of glaciation [Imbrie et al., 1992]. By comparison between the paleoclimatic record and the nonlinear stochastic model, it is demonstrated that the record can be generated by the forcing from insolation changes due to the obliquity cycle through the full record including the last $1 \mathrm{Ma}$. It has been long known that the 100 ka world is not linearly responding to the orbital forcing [Kominz and Pisias, 1979], but even in the $41 \mathrm{ka}$ world the climate response to the orbital forcing is nonlinear [Ashkenazy and Tziperman, 2004]. The assumption here is that the orbital forcing resulted in periodic jumps between two stable climate states. What happened approximately $800 \mathrm{ka}$ to $1 \mathrm{Ma}$ ago was that a third deep glacial state became accessible resulting in a change in length of the glacial cycles. The reason for this midPleistocene transition (MPT) is unknown, and attributed to a gradual cooling due to a decreasing atmospheric $\mathrm{CO}_{2}$ level [Saltzman and Maasch, 1991] or a change in the bedrock erosion (the regolith hypothesis) [Clark and Pollard, 1998]. For a review see Clark et al. [2006].

[6] For a simple zero-dimensional model of the Earth, where the climate is characterized by one number, the global mean temperature, there is ambiguity in ascribing the orbital forcing from the time and space varying insolation field across the globe.

[7] In a very interesting series of papers Huybers and Wunsch [2005] and Huybers and Tziperman [2008] argue that integrated summer insolation is the relevant measure of orbital forcing. This is closely related to the concept of degree days, which is the annual number of days with 
temperatures above freezing. The underlying assumption being that ablation from the glaciers is a dominant climate driver. This measure is dominated by the obliquity cycle since, as noted by Huybers [2006], the increased insolation when Earth is close to the sun in its orbit is compensated by shorter time spent there because of Kepler's second law. Thus the total insolation during the degree days becomes independent on the precessional cycle. Here we shall assume the integrated insolation exceeding $200 \mathrm{~W} / \mathrm{m}^{2}$ to be a proxy for the degree days forcing.

[8] In contrast to this Paillard [1998] shows, using a simple rule-based model of jumping between three different quasi-stationary climate states, that the climate record can be a response to the summer solstice insolation at $65 \mathrm{~N}$. The two proposed forcings (degree day insolation and 26 June insolation) are different, since the latter has a strong component of the precessional cycle. Using the 26 June insolation as the better proxy for the forcing can be rationalized from the point of view of a threshold crossing dynamics, since the extremal values (midsummer insolation) would then be the governing parameter. However, since we cannot decide between the two within the framework of a simple model, we shall take the alternative approach of assuming the linear combination of the two, considered as a first-order expansion, which gives the best fit between the observed record as response to the forcing.

[9] The starting point for the model is the rule-based model proposed by Paillard [1998]. The rules imposed by Paillard will be derived from an effective governing stochastic differential equation. This gives a dynamical explanation of the rule-based model and potentially narrow the range of possible climate mechanisms and models capable of explaining the glacial cycles.

\section{Nonlinear Climate Response to the Forcing}

[10] Because of the high dimensionality and the stochastic nature of the climate fluctuations it is highly unlikely that regular periodicities can result from internal oscillatory modes alone. It is much more plausible that nonlinear responses to weak external periodic forcing would lead to periodic behavior. There is evidence from observations as well as models that multiple states exist in the climate system [Imbrie et al., 1992]. This suggests a possible scenario of periodically induced destabilizations of quasistable climate states.

[11] The multiple states governed by ice albedo feedback as proposed in simple energy balance models [Sellers, 1969; Bodyko, 1969] have been demonstrated in a more realistic climate model [Langen and Alexeev, 2004]. Likewise it has been demonstrated that more realistic ocean models [Rahmstorf, 1995] has a structure of stability and bifurcation points similar to the simple box models [Stommel, 1961]. It is thus plausible that within the high dimensional climate system there are slow manifolds for which bifurcation points exist [Broecker, 1997].

[12] Bifurcation points in the system describe structural changes in the stability of metastable states as a function of a control parameter measuring the forcing of the system. The validity of linear analysis near the stable states permits a complete classification of the possible types of bifurcation points in any nonlinear system [Guckenheimer and Holmes, 1986].

[13] The paleoclimatic records, especially from deep sea cores and ice cores, show that at least three distinct climate states have been identified [Imbrie et al., 1992]. These are (1) a deep glacial state for which the North American and Fennoscandian ice sheets had their full extend and the Northern Atlantic deep water formation was weakened because of the extended sea ice cover; however, more recent analysis of sediment records show that even in the deep glacial state there was a substantial thermohaline flow in the North Atlantic [McManus et al., 2004]; (2) an intermediate or preglacial state where the ice sheets were in a slow build up phase and the Gulf Stream made way for some intermediate water formation and the boreal heat pump; and (3) the interglacial state where the northern ice sheets, except from Greenland, were gone and the deep water formation occurred as today north of Denmark straight with the Nordic heat pump. The fourth state described by Imbrie et al. [1992] will be regarded as a transition state here.

[14] The three states can be identified with the ones labeled $G$ (deep glacial), $g$ (preglacial), and $i$ (interglacial), respectively, by Paillard [1998]. Here we adapt the same notation. Paillard observed in the paleoclimatic record that there seems to be "forbidden" transitions between the three states. In the period 2-1 Ma B.P. the record shows regular oscillations between only the two states $i$ and $g$, while in the period 1-0 Ma B.P. there is only a specific sequence of occurrences: $i \rightarrow g \rightarrow G \rightarrow i$ permitted. The model presented here gives a dynamical explanation of this observation.

\section{Stochastic Model}

[15] The model, which is empirical, assumes that the climate dynamics is reflected in a single variable $x(t)$. This is as usual taken to be (minus) the global ice volume, represented by the deep sea oxygen isotope ratio, roughly proportional to global mean surface temperature anomaly. The dynamics is described by an effective nonlinear stochastic differential equation,

$$
d x=f_{\alpha}(x, \mu) d t+\sigma d B
$$

where the white noise term $\sigma d B$ with intensity $\sigma$ describes the influence of the nonresolved variables and the internally generated chaotic climate fluctuations. It is within this framework the roles of the orbital forcing and internal stochastic forcing is investigated. The deterministic part, $f_{\alpha}(x, \mu)$, of the dynamics depends on the external orbital forcing, labeled by a single control parameter $\mu$ and internal parameters, represented by $\alpha$. Note that equation (1) is nonautonomous, since $\mu$ and $\alpha$ are time-dependent.

[16] The full climate dynamics can obviously not be completely reconstructed by such a single valued function. However, since stability and bifurcations are topological quantities it could be robust with respect to the detailed dynamics modeled. It is thus the bifurcation structure of $f_{\alpha}(x, \mu)$, with respect to the control parameter $\mu$, which determines the climate development. 

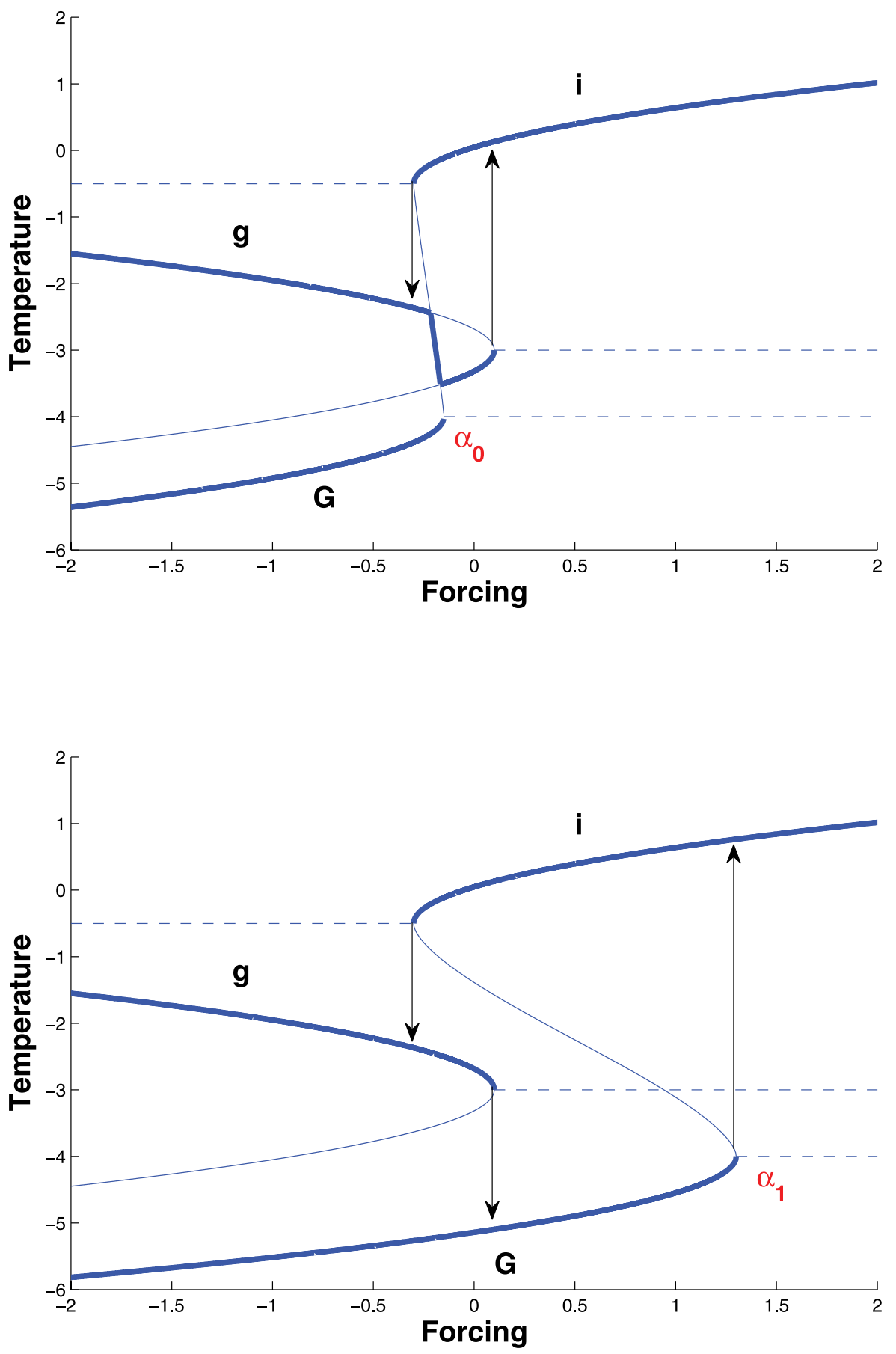

Figure 1. The bifurcation diagram for the model. Along the $x$ axis is the forcing represented by the control parameter $\mu$, and along the $y$ axis are the fixed points $\left\{x_{0}(\mu) \mid f\left(x_{0}, \mu\right)=0\right\}$ of the drift function $f_{\alpha}(x, \mu)$. The drift function is simply approximated by a fifth-order polynomial, with the roots determined by the fixed points. The horizontal dashed line segments indicate (real part of) sets of complex conjugate roots. The thick curves show the stable fixed points. The bifurcation point $\alpha$ is the point where the deep glacial state $G$ disappears. The arrows indicate the hysteresis loop as the forcing parameter is changed. (top) The glacial state $G$ is not accessible. (bottom) Now the location of the bifurcation point $\alpha$ has changed in such a way that the deep glacial state $G$ is accessible.

[17] Guided by the observed record and the transition rules proposed by Paillard [1998] we can empirically construct a bifurcation diagram: Figure 1 (top) shows the bifurcation diagram for the drift function $f_{\alpha}(x, \mu)$ as a function of $\mu$ at the time interval 2-1 Ma B.P. The bifurcation diagram shows the curves $\left\{x_{0}(\mu) \mid f\left(x_{0}, \mu\right)=0\right\}$. The thick curves are the stable fixed point curves for which $\partial_{x} f<0$, while the thin curves are the unstable fixed 
(a)

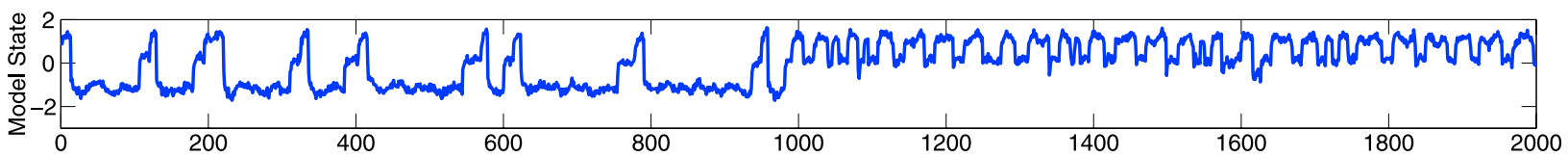

(b)

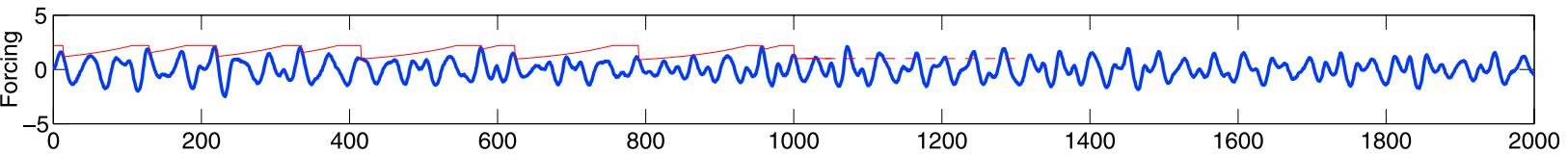

(c)

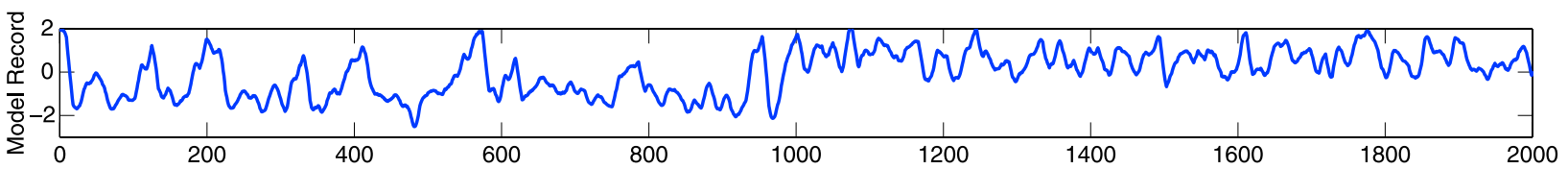

(d)

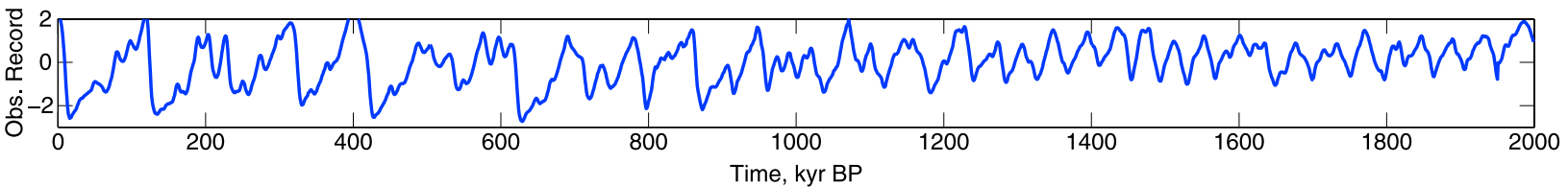

Figure 2. (a) A realization of the model. (b) The orbital forcing driving the model. The red curve shows $\alpha(t)$, where the jumps to $\alpha=\alpha_{1}$ are triggered by the transition $G \rightarrow i$. The next transition is in the low noise limit only possible when the blue curve is above the red curve. The dashed line represents the initial level of $\alpha_{1}$ sufficiently small to prevent the transition $g \rightarrow G$. (c) A "pseudopaleorecord," where a red noise component representing the nonclimatic noise is added to the model realization in Figure $2 \mathrm{a}$. (d) The (normalized) paleoclimatic isotope record from a composite of ocean cores. The record is a proxy for the global sea level or minus the global ice volume. See text for more explanations.

point curves for which $\partial_{x} f>0$. Thus in the case of no additional noise $(\sigma=0$ in equation (1)) the state of the system $x(t)$ is uniquely determined from the initial state $x(0)$ and the development of the forcing $\mu(t)$.

[18] In the real climate system the internal noise is substantial and the system will not reside exactly in the steady states determined by the bifurcation diagram. Thus the full drift function needs to be parametrized. The simplest way to parametrize the drift function in accordance with the bifurcation diagram is as a fifth-order polynomial:

$$
f_{\alpha}(x, \mu)=\Pi_{j=1}^{5}\left(x-x_{\alpha}^{j}(\mu)\right),
$$

where $x_{\alpha}^{j}(\mu)$ is the $j$ th steady state (zero points) in the bifurcation diagram. As labeled in Figure 1 the parameter $\alpha$ determines the position of the lower bifurcation point. See Appendix A for more details. A Matlab code of the model is available in the auxiliary material. ${ }^{1}$

[19] It should be noted that this is, of course, not the only possible drift function corresponding to this bifurcation diagram. In order to reconstruct the drift function from the observed realization, one could in principle obtain the stationary probability density $p_{\mu_{0}}(x)$ by sorting $x(t)$ according to $\mu(\mathrm{t})=\mu_{0}$. Assuming that $\mu(t)$ is changing slowly in comparison to the timescale for $x(t)$ to drift to a stationary state $x_{0}\left(f_{\alpha}\left(x_{0}, \mu\right)=0\right)$, one could then obtain $f_{\alpha}\left(x, \mu_{0}\right)$ by solving the Fokker-Planck equation [Gardiner, 1985] associated with equation (1) for fixed $\mu=\mu_{0}$. This would require

\footnotetext{
${ }^{1}$ Auxiliary materials are available in the HTML. doi:10.1029/ 2008PA001673.
}

a very long data series and complete absence of additional nonclimatic noise in the proxy data. This is not the case for the existing paleoclimatic record.

[20] The climate forcing is, as mentioned before, taken to be a linear combination of the summer solstice $65 \mathrm{~N}$ insolation $\left(f_{s s}\right)$ and the integrated summer insolation at $65 \mathrm{~N}\left(\bar{f}_{I}\right)$, where the summer period is defined as the period where the daily mean insolation exceeds $I=200 \mathrm{~W} / \mathrm{m}^{2}$. The model results are robust with respect to the threshold $I$ chosen in a rather broad interval. The forcing, $f=\lambda \bar{f}_{I}+(1-\lambda) f_{s s}$, shown in Figure $2 \mathrm{~b}$ is calculated using the code provided by Huybers and Eisenman [2006]. Values of $\lambda$ around 0.5 gives the best result, $\lambda=0.5$ is used. The $f_{s s}$ component has a relatively strong precessional component which is absent in $\bar{f}_{I}$. For small values of $\lambda$ there is an overwhelming probability of the last glacial termination to occur $41 \mathrm{ka}$ prior to what is observed. Thus the assignment $\lambda \approx 0.5$ might, within the framework of the nonlinear model, be interpreted as an empirical determination of the dominating components of the orbital forcing.

\section{Hysteresis Behavior}

[21] The diagram in Figure 1 (top) shows the fixed points of $f_{\alpha}(x, \mu)$ as a function of the deterministic forcing $\mu$. The three branches of stable fixed points $x^{j}(\mu)$ for the function, such that $f_{\alpha}\left(x^{j}, \mu\right)=0$ and $\partial_{x} f_{\alpha}\left(x^{j}, \mu\right)<0$, are indicated by thick curves. The functional form for the five functions $x^{j}(\mu)$ are given in Appendix A. The specification of the $x^{j}(\mu)$ 's and equations (1) and (2) completely defines the model. Since $x$ is a proxy for global mean surface temper- 
ature anomaly (or minus global ice volume), the lower branch corresponds to the deep glacial state $G$. The middle branch corresponds to the climate state $g$ and the upper branch to the interglacial state $i$. The thin curves correspond to the separating unstable fixed points. The dashed line segments correspond to pairs of complex conjugate roots in the fifth-order polynomial. Note again that assuming a polynomial drift function, this is uniquely determined from the roots, except from a trivial multiplicative constant.

[22] Suppose now that the climate is in either of the states $g$ or $i$ and the climatic noise is too weak to induce a crossing of a barrier separating the stable states. Then the only way a forcing induced shift between the climate states can occur is through bifurcations and a hysteresis as sketched by the arrows. Clearly the climate state $G$ is unreachable.

[23] Assume now that the lower bifurcation point, indicated by $\alpha_{0}$ in Figure 1 (top) moves toward larger values of $\mu$ indicating that a stronger forcing is needed in order to destabilize the deep glacial state. In this case, $\alpha_{0} \rightarrow \alpha_{1}$ shown in Figure 1 (bottom), the glacial state $G$ is now reachable and a hysteresis loop $i \rightarrow g \rightarrow G \rightarrow i$ will appear. The central postulate of the model is the change in this bifurcation structure represented by the shift of the point $\alpha$ (from $\alpha_{0}$ to $\alpha_{1}$ on the $\mu$ axis) at the mid-Pleistocene transition. This constitutes a dynamical explanation for the rule-based model by Paillard [1998].

[24] The change in the position of the lower bifurcation point is modeled so that $\alpha=\alpha_{1}$ when the climate is in state $i$. When the state $G$ is reached through two bifurcations, $\alpha$ starts to decrease with time. This gradual change in the bifurcation diagram is modeled as a relaxation, $d \alpha / d t=$ $-\left(\alpha-\alpha_{0}\right) / \tau$, where $\alpha_{0}$ is the early Pleistocene equilibrium value and $\tau$ is a relaxation time. When the climate bifurcates through the rapid transition $G \rightarrow i$, the parameter $\alpha$ again change to $\alpha_{1}$.

[25] In order for the climate to skip the $41 \mathrm{ka}$ obliquity pacing of deglaciations the timescale $\tau$ governing the bifurcation structure must be considerably longer than $41 \mathrm{ka}$. The model results are quite insensitive to the specific value of $\tau$ in the interval $70-130 \mathrm{ka}$, and is set to be $100 \mathrm{ka}$. It is a major challenge to interpret the behavior of the bifurcation point $\alpha$, governed by such a long timescale in terms of real climate dynamics. One could speculate that it is linked to the carbon cycle and with erosion of continents on these long timescales.

\section{Comparison Between the Paleoclimatic Record and the Model}

[26] The presence of the stochastic forcing implies that the climate evolution is not fully deterministic.

[27] Figure 2a shows a particular realization of the model. Figure $2 b$ shows the forcing, and the red curve shows the value of $\alpha$, which is defined as the position of the lower bifurcation point in Figure 1 along the axis of the forcing (the $x$ axis). Note that a transition $G \rightarrow i$ without noise assistance is only possible when the forcing exceeds the value of alpha (that is when the blue curve is above the red curve in Figure 2b).
[28] The composite Atlantic ocean sedimentation record for the period 0-2000 ka B.P. generated by Huybers [2007] is shown in the Figure $2 \mathrm{~d}$. The record is the benthic oxygen isotope sequence. The curve is plotted with normalized variance and the mean subtracted. This is a proxy for the global ice volume. The dating is based on a depth-age model independent from astronomical tuning. Note that the model resides predominantly in the deep glacial state $G$, rather than the preglacial state $g$. This is opposite to the model by Paillard [1998].

[29] The differences between the single records compositing the stacked record gives an estimate of the additional noise from bioturbation and other factors that makes the record different from a true record of ice volume. So in order to compare the model with the observed climate record an additional red noise, of the same magnitude as the difference in deep sea records is added to the model. This is shown in Figure 2c, which should be compared with the observed record in Figure 2d.

\section{Role of the Stochastic Noise}

[30] In order to investigate the role of the stochastic noise a set of realizations of the model are presented in Figure 3. For the comparison between the model and the proxy climate records we focus on the rapid transitions $G \rightarrow i$, called terminations [Broecker, 1984; Raymo, 1997]. Figure 3a shows a realization with no stochastic noise. This is the deterministic climate response to the orbital forcing. It is seen that the last five terminations are reproduced as observed (Figure 2d), but there are fewer interglacials in the earlier part of the late Pleistocene period (1000-500 ka B.P.) than in the observed record. From Figure $2 b$ it is seen that the amplitude of the orbital forcing is low in this period. Figures $3 \mathrm{~b}-3 \mathrm{e}$ show different realizations with a moderate stochastic forcing $(\sigma=0.8 \mathrm{~K} / \sqrt{\mathrm{ka}})$. In these realizations the added noise induces additional terminations at different times, suggesting a fundamental unpredictability in glacial terminations. With a larger intensity stochastic forcing the terminations tend to occur at more obliquity cycles, corresponding to $41 \mathrm{ka}$ ice ages, contrary to the observations for the late Pleistocene record.

[31] Even though the observed ice age history is not expected to be reproduced in a given model realization, the spectral density is similar. The spectral signature of the change in the climatic record from the $41 \mathrm{ka}$ world $(2-1 \mathrm{Ma}$ B.P.) to the $100 \mathrm{ka}$ world (1-0 Ma B.P.) is shown in Figure 4. The spectral signature is well reproduced in the randomly chosen model realization.

\section{Suggestive Interpretation of the Dynamics}

[32] This section may be skipped without loss of continuity. The model is empirically derived from the paleoclimatic record. Since the record is only a one dimensional representation of the climate state and the high dimensional real climate variability is described as noise in an effective stochastic governing equation, the interpretation of the underlying dynamics can only be suggestive.

[33] The climate stability can be described in terms of temperature-albedo feedbacks or equivalently ice volume- 


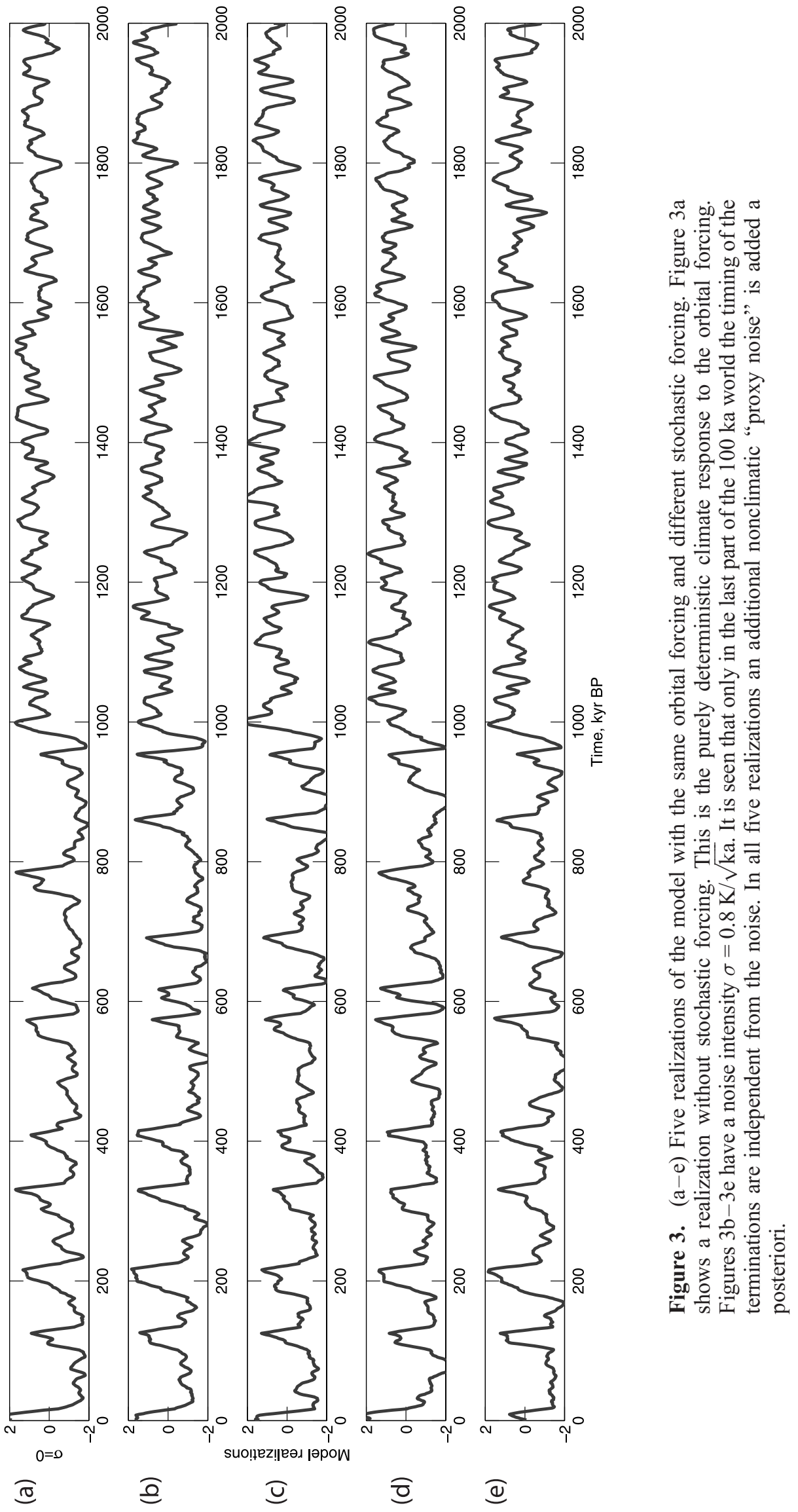



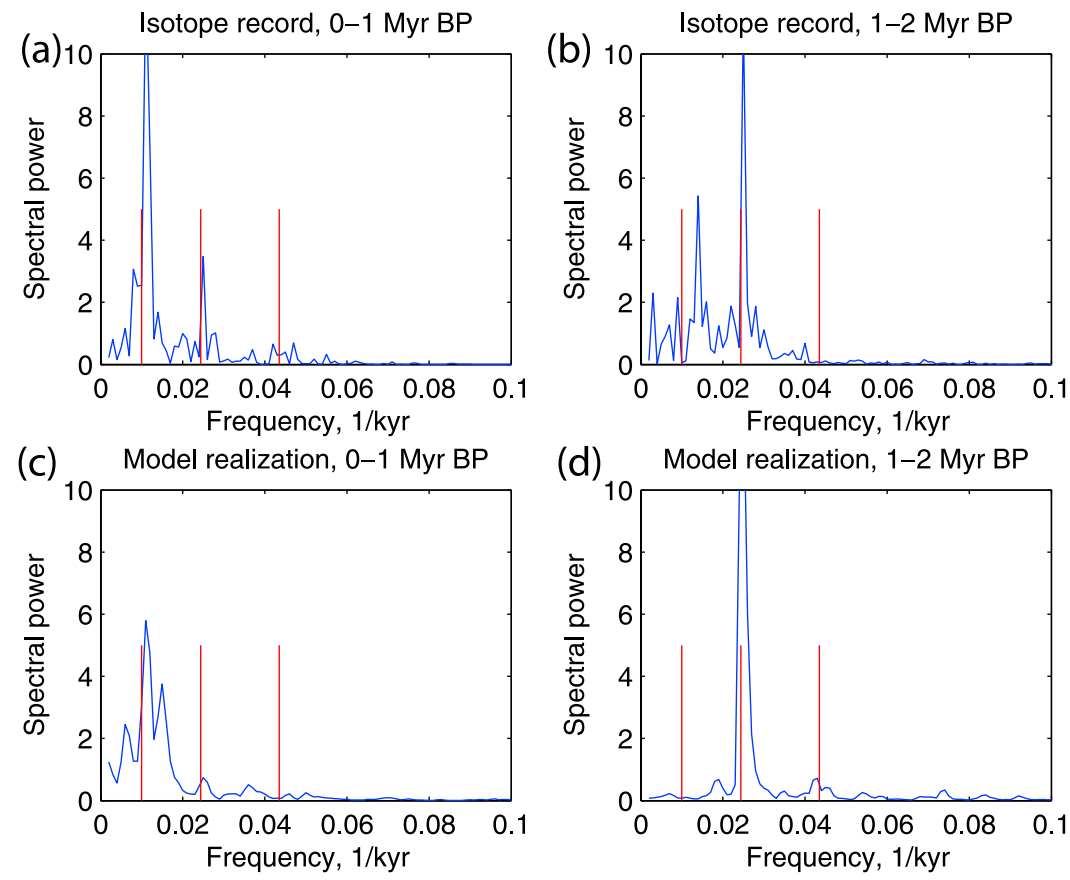

Figure 4. The spectral power of the sediment records for the two periods (a) 0-1 Ma and (b) 1-2 Ma. The red markers indicate 100, 41, and $23 \mathrm{ka}$ periods. (c and d) The corresponding spectra for the randomly chosen realization of the model shown in Figure 3. Both the climate record and the model show a transition from the $41 \mathrm{ka}$ world to the $100 \mathrm{ka}$ world.

precipitation feedbacks [North et al., 1981; Ghil and Childress, 1987; Tziperman and Gildor, 2003]. From the latter perspective we may write the nonlinear drift $f_{\alpha}(x, \mu)$ equals ablation (ice melt off) minus accumulation (precipitation on ice sheets). In Figure 5 the bifurcation diagrams for the two periods are repeated. Figures $6 a-6 f$ show graphs of the accumulation and ablation curves along the transects shown in Figure 5. Going from top to bottom corresponds to increasing orbital forcing.

[34] The ablation, $f_{\alpha}^{a b l}=c_{0}+c_{1} \cdot\left(x-T_{m}\right)$, is assumed to be a linearly increasing function of the temperature. The constants $c_{0}, c_{1}$ and the effective temperature of melting $T_{m}$ are related to the specific heat of melting, relation between global temperature and high-latitude temperatures et cetera. It could potentially be estimated from paleo-observations of freshwater influx into the oceans. The accumulation, $f_{\alpha}^{a c c}$, is a more complex nonlinear function of temperature. The accumulation is empirically determined from (2) simply as $f_{\alpha}^{a c c}=f_{\alpha}^{a b l}-f_{\alpha}$. This splitting of the drift function should be considered schematic, and attempt toward more realism would involve much more complex climate models, which shall not be pursued here. The stable fixed points for $f_{\alpha}(x, \mu)$ with respect to $x$ are marked with red circles in Figures $6 \mathrm{a}-6 \mathrm{f}$. The accumulation depends on the hydrological cycle, sea ice cover and snow to rain ratio. Furthermore, it could depend on the temperature of the deep ocean through the sea ice shift (SIS) mechanism suggested by Gildor and Tziperman [2000] and Tziperman and Gildor [2003]. This is a good candidate for explaining the difference between the early Pleistocene (Figures 6d-6f) and the late Pleistocene (Figures $6 \mathrm{a}-6 \mathrm{c}$ ) climates. The major dif- ference is seen by comparing Figures $6 \mathrm{~b}$ and $6 \mathrm{e}$. Figure $6 \mathrm{~b}$ represents the climate drift in the late Pleistocene period where the deep ocean is cold [Ruddiman et al., 1989]. The low temperature of the deep ocean effectively prevents heat exchange with the upper water masses, such that an extensive sea ice cover can develop [Tziperman and Gildor, 2003]. The barrier separating the states $G$ and $g$ could be caused by a change in the hydrological cycle such that to the left of the barrier (toward state $G$ ) the accumulation decreases rapidly with temperature because of the growth of sea ice cover, while to the right of the barrier (toward state $g$ ) the accumulation decrease with increasing temperature. This could be due to the southward position of the summer polar front changing the precipitation pattern over the ice sheets. Perhaps this would result in more rainfall and less snow fall over the ice sheets as temperature increases leading to the decreasing accumulation with temperature [Tziperman and Gildor, 2003].

[35] However, the real climate has not, except perhaps for short transient periods, been in such a state, thus it cannot be reconstructed from paleoclimatic evidence and we will have to rely on physical reasoning and future realistic climate models for explanations. In the early Pleistocene period for the same forcing the barrier is absent (Figure 6e).

[36] When the state $i$ is reached, the Antarctic ice core records show that the level of atmospheric $\mathrm{CO}_{2}$ has increased because of oceanic heating and following $\mathrm{CO}_{2}$ release. When the climate state $G$ is reached after two bifurcations the atmospheric $\mathrm{CO}_{2}$ concentration will gradually decrease as the ocean cools. Furthermore, the large glaciers build up on land. 

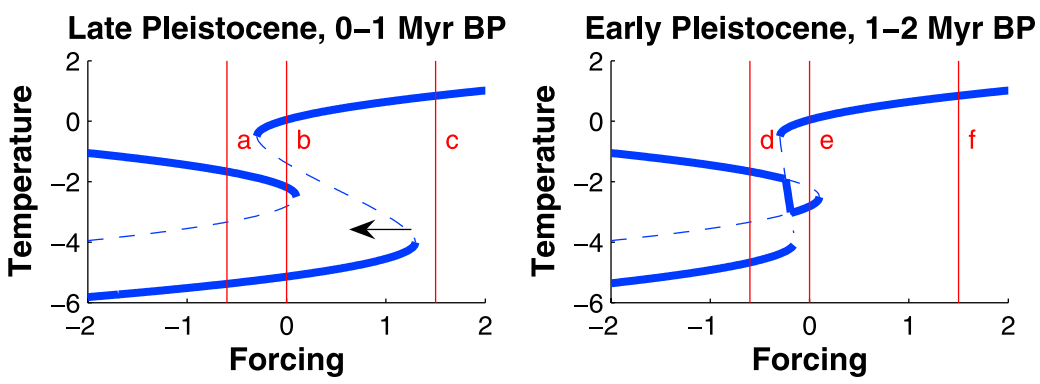

Figure 5. The form of the drift function as a function of the orbital forcing.

[37] In the late Pleistocene period when the glacial state $G$ is reached the meridional heat transport in the oceans is strongly reduced. This would trap the warm ocean waters in the tropics, leading to a slow heating of the deep tropical ocean. This would perhaps in turn lead to an oceanic climate more similar to the one in the early Pleistocene period, where the deep ocean was warmer [Tziperman and Gildor, 2003]. This could then imply the slow change of the position of the bifurcation point $\alpha$ in the direction of its position during the early Pleistocene period. This would make way for the orbital forcing to trigger the transition $G \rightarrow i$. The triggering of a transition is then a combination of the deterministic orbital forcing and the internal stochastic noise induced forcing.

[38] The forcing will trigger the transition $G \rightarrow i$ way before the early Pleistocene value is reached. One could speculate that the slow heating of the tropical ocean during the state $G$ could also be related to the lower atmospheric
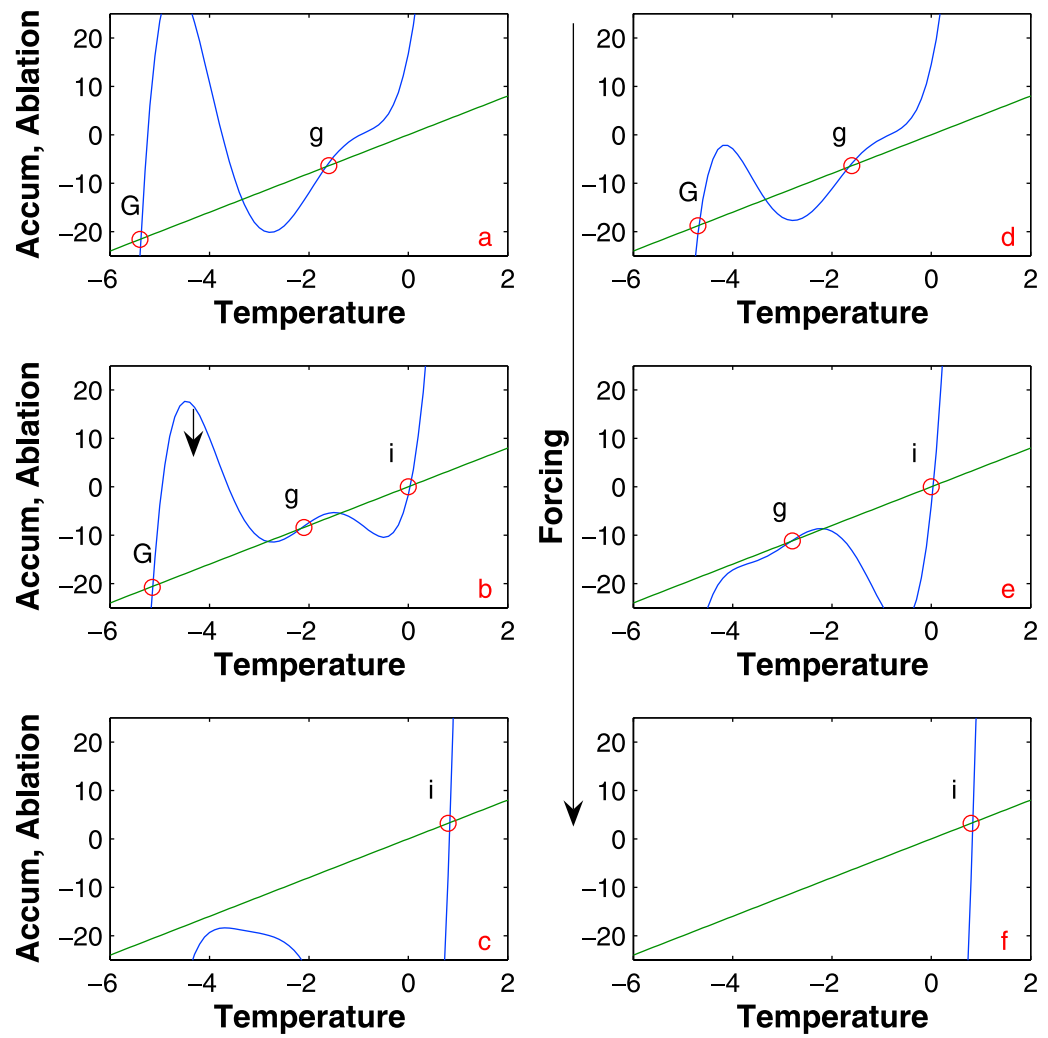

Figure 6. $(\mathrm{a}-\mathrm{c})$ The late Pleistocene graphs correspond to the vertical intersections in Figure 5 (left). $(d-f)$ The early Pleistocene graphs correspond to the intersections in Figure 5 (right). The major difference between the late Pleistocene and early Pleistocene periods is seen by comparing Figures $6 \mathrm{~b}$ and 6e corresponding to the same orbital forcing in the two periods. When the climate is in the glacial state $\mathrm{G}$, there will be a slow lowering of the barrier separating $G$ and $g$ indicated by arrows in Figures 5 (left) and $6 \mathrm{~b}$. The (arbitrary) splitting of the drift function into an ablation part and an accumulation part is only suggestive. 


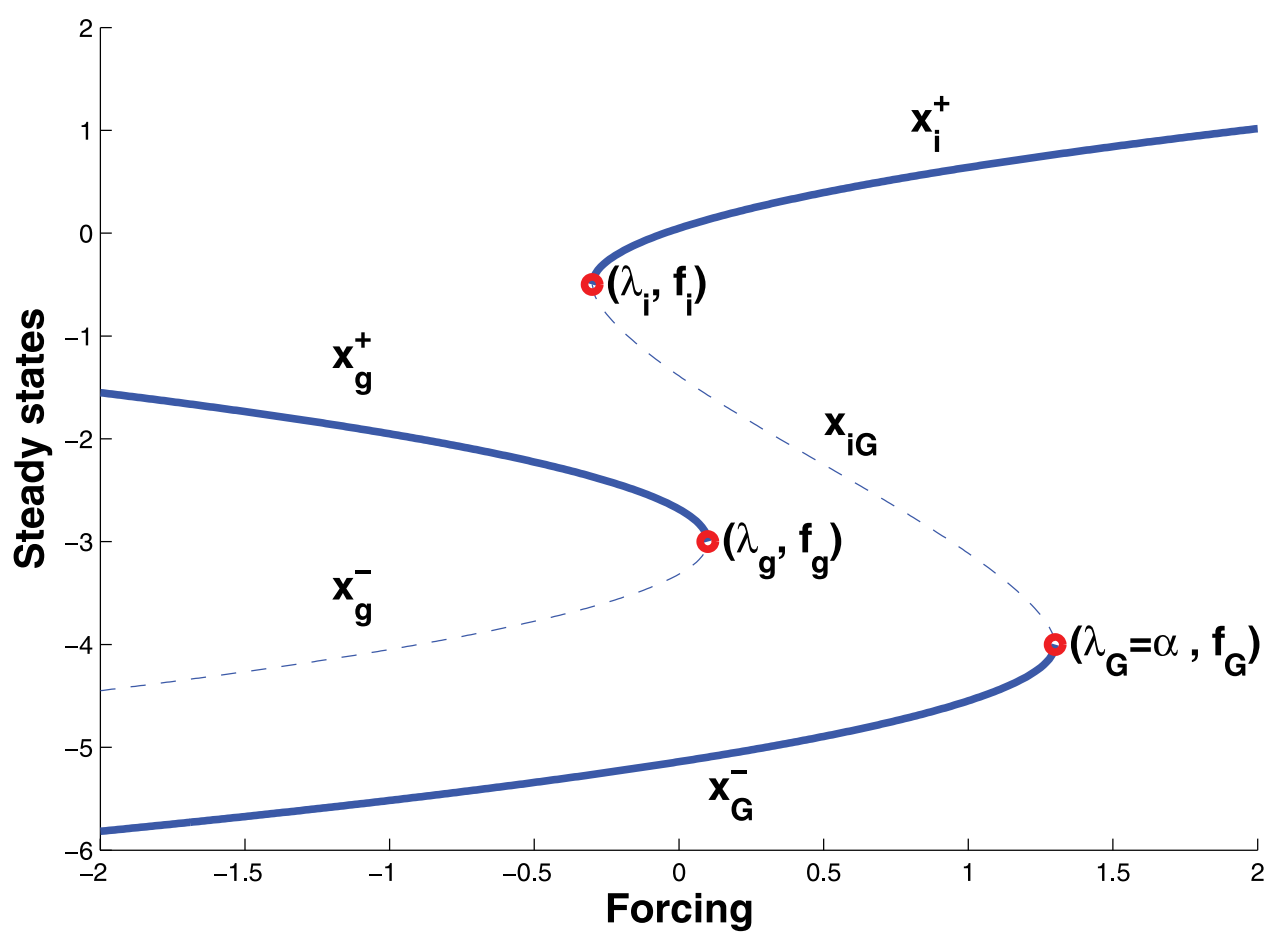

Figure A1. The bifurcation diagram with stable branches $x_{i}^{+}, x_{g}^{+}$, and $x_{G}^{-}$. The three bifurcation points are $(\mu, x(\mu))=\left(\lambda_{i}, f_{i}\right)$ where the interglacial state disappears, $\left(\lambda_{g}, f_{g}\right)$ where the intermediate glacial state disappears, and, finally, $\left(\lambda_{G}, f_{G}\right)$ where the deep glacial state disappears. See text for explanation.

$\mathrm{CO}_{2}$ concentration in the late Pleistocene period [Maasch and Saltzman, 1990; Saltzman, 1990]. However, one should stress that interpretation in terms of accumulation, ablation and ocean circulation is suggestive. Only the bifurcation structure of the governing equation is obtained from the paleoclimatic records. Future realistic climate models with the same type of bifurcation structure are necessary to substantiate these speculations.

\section{Summary}

[39] In summary the empirical stochastic model presented supports the suggestion that the transition from the $41 \mathrm{ka}$ world to the $100 \mathrm{ka}$ world occurring approximately $1 \mathrm{Ma}$ to $800 \mathrm{ka}$ B.P. is due to a structural change in the bifurcation diagram describing the stability of the system as a function of the forcing. The glacial cycles are not solely a deterministic response to the orbital cycles. The internal noise also plays a role in triggering the jumps between the different climatic states in the first part of the $100 \mathrm{ka}$ world, this makes the ice ages fundamentally unpredictable. Currently the state-of-the-art general circulation climate models are far from being able to simulate the observed glacial climate variations. It is even not known if they possess a nontrivial bifurcation structure. This lack of dynamical range might be due to underestimation of internal variability in too coarse resolution, thus the climate noise is to weak to induce transitions from one stable climate state to another. The identification of the dynamical bifurcation diagram from observations, should be a guideline for identification of physical mechanisms and ultimately for building realistic glacial climate models.

\section{Appendix A: Parametrizing the Bifurcation Diagram}

[40] The fixed point curves in the hysteresis diagram determines the drift function $f_{\alpha}(x, \mu)$. The curve for the interglacial stage " $i$ " is modeled as the parabolic curve

$$
\left(x_{i}(\mu)-f_{i}\right)^{2}=\mu-\lambda_{i} \Rightarrow x_{i}^{ \pm}(\mu)=f_{i} \pm \sqrt{\mu-\lambda_{i}} .
$$

Thus its bifurcation point is specified by the cartesian coordinates $\left(\lambda_{i}, f_{i}\right)$, and from the data it is assessed as $\left(\lambda_{i}\right.$, $\left.f_{i}\right)=(-0.3,-0.5)$ (see Figure A1). For $\mu<\lambda_{i}$ there are two complex conjugate roots not corresponding to real fixed points. These are indicated by dashed lines in Figure 1. For $\mu>\lambda_{i}$ there are two branches (a stable and an unstable) of fixed points.

[41] Similarly the curve for the intermediate glacial state " $g$ " and the curve for the deep glacial state " $G$ " are modeled as parabolic curves of the form

$$
(x(\mu)-f)^{2}=\lambda-\mu,
$$

i.e.,

$$
x_{g}^{ \pm}(\mu)=f_{g} \pm \sqrt{\lambda_{g}-\mu},
$$


with the bifurcation point assessed from the data to $\left(\lambda_{g}, f_{g}\right)=$ $(0.1,-3.0)$, and

$$
x_{G}^{ \pm}(\mu)=f_{G} \pm \sqrt{\lambda_{G}-\mu},
$$

with the bifurcation point set to $\left(\lambda_{G}, f_{G}\right)=(\alpha(t),-4.0)$. The parameter $\alpha(t)$ thus determines the horizontal position of the lower bifurcation point in Figure A1. Now, as seen in Figure A1, only the upper (stable) branch, $x_{i}^{+}$, and the lower (stable) branch, $x_{G}^{-}$, are plotted. The curve connecting the two bifurcation points is simply given as a linear interpolation between the two unstable branches:

$$
x_{i G}=\lambda x_{i}^{-}+(1-\lambda) x_{G}^{+},
$$

where $\lambda=\left(\mu-\lambda_{G}\right) /\left(\lambda_{i}-\lambda_{G}\right)$ and $\lambda_{i} \leq \mu \leq \lambda_{G}$. By this the five fixed points are now defined and the drift function is

$f_{\alpha}(x, \mu)=-\left(x-x_{g}^{+}\right)\left(x-x_{g}^{-}\right)\left(x-x_{i}^{+}\right)\left(x-x_{G}^{-}\right)\left(x-x_{i G}\right)$.

The first minus sign ensures that $f>0$ for $x \rightarrow-\infty$ and $f<0$ for $x \rightarrow \infty$ as it should. This is the form specified in equation (2). The parameter $\alpha(t)$ is specified from the climate state. If the climate is in the interglacial state the parameter is set to $\alpha=\alpha_{1}$. When the climate jumps to the glacial state " $G$," the parameter change by an exponential decay to $\alpha_{0}$ with timescale $\tau$ :

$$
\frac{d\left(\alpha-\alpha_{0}\right)}{d t}=-\frac{\left(\alpha-\alpha_{0}\right)}{\tau} .
$$

The parameters used here are $\left(\tau, \alpha_{0}, \alpha_{1}\right)=\left(10^{5}\right.$ years, $0.5,2.2)$.

[42] Acknowledgment. This research was supported in part by the National Science Foundation under grant PHY05-51164.

\section{References}

Ashkenazy, Y., and E. Tziperman (2004), Are the 41 kyr oscillations a linear response to Milankovitch forcing?, Quat. Sci. Rev., 23, 1879-1890.

Ashkenazy, Y., D. R. Baker, and H. Gildor (2005), Simple stochastic models for glacial dynamics, J. Geophys. Res., 110, C02005, doi:10.1029/2004JC002548.

Benzi, R., G. Parisi, A. Sutera, and A. Vulpiani (1982), Stochastic resonance in climate change, Tellus, 34, 10-16.

Bodyko, M. I. (1969), The effect of solar radiation variations on the climate of the Earth, Tellus, 21, 611-619.

Broecker, W. S. (1984), Terminations, in Milankovitch and Climate: Understanding the Response to Astronomical Forcing, NATO ASI Ser., Ser. C, vol. 126, edited by A. Berger et al., pp. 687-698, D. Reidel, Dordrecht, Netherlands.

Broecker, W. S. (1997), Thermohaline circulation, the Achilles heel of our climate system: Will man-made $\mathrm{CO}_{2}$ upset the current balance?, Science, 278, 1582-1588.

Clark, P. U., and D. Pollard (1998), Origin of the middle Pleistocene transition by ice sheet erosion of regolith, Paleoceanography, 13, 1-9.

Clark, P. U., D. Archer, D. Pollard, J. D. Blum, J. A. Rial, V. Brovkin, A. C. Mix, N. G. Pisias, and M. Roy (2006), The middle Pleistocene transition: Characteristics, mechanisms, and implications for long-term changes in atmospheric $\mathrm{pCO}_{2}$, Quat. Sci. Rev., 25, 3150-3184.

Gardiner, C. W. (1985), Handbook of Stochastic Methods, Springer, New York.

Ghil, M., and S. Childress (1987), Topics in Geophysical Fluid Dynamics: Atmospheric Dynamics, Dynamo Theory, and Climate Dynamics, Springer, New York.

Gildor, H., and E. Tziperman (2000), Sea ice as the glacial cycles' climate switch: Role of seasonal and orbital forcing, Paleoceanography, $15,605-615$.

Guckenheimer, J., and P. Holmes (1986), Nonlinear Oscillations, Dynamical Systems, and Bifurcations of Vector Fields, Springer, New York.
Huybers, P. (2006), Early Pleistocene glacial cycles and the intergrated summer insolation forcing, Science, 313, 508-511.

Huybers, P. (2007), Glacial variability over the last two million years: An extended depthderived agemodel, continuous obliquity pacing, and the Pleistocene progression, Quat Sci. Rev., 26, 37-55.

Huybers, P., and I. Eisenman (2006), Integrated summer insolation calculations, Data Contrib. 2006-079, http://www.ncdc.noaa.gov/paleo/ forcing.html, World Data Cent. for Paleoclimatol., Boulder, Colo.

Huybers, P., and E. Tziperman (2008), Integrated summer insolation forcing and 40,000-year glacial cycles: The perspective from an icesheet/energy-balance model, Paleoceanography, 23, PA1208, doi:10.1029/2007PA001463.

Huybers, P., and C. Wunsch (2005), Obliquity pacing of the late Pleistocene glacial terminations, Nature, 434, 491-494.

Imbrie, J., et al. (1992), On the structure and origin of major glacial cycles: 1. Linear responses to Milankovitch forcing, Paleoceanography, 7, $701-738$.

Imbrie, J., et al. (1993), On the structure and origin of major glacial cycles: 2 . The $100,000-$ year cycle, Paleoceanography, 8, 699-735.

Källen, E., C. Crafoord, and M. Ghil (1979), Free oscillations in a climate model with icesheet dynamics, J. Atmos. Sci., 36, $2292-$ 2303.

Kominz, M. A., and N. G. Pisias (1979), Pleistocene climate: Deterministic or stochastic, Science, 204, 171-172.

Langen, P. L., and V. A. Alexeev (2004), Multiple equilibria and asymmetric climates in the CCM3 coupled to an oceanic mixed layer with thermodynamic sea ice, Geophys. Res. Lett., 31, L04201, doi:10.1029/2003GL019039.

LeTreut, H., and M. Ghil (1983), Orbital forcing, climate interactions, and glacial cycles, J. Geophys. Res., 88, 5167-5190.

Maasch, K., and B. Saltzman (1990), A low-order dynamical model of global climate varia- bility over the full Pleistocene, J. Geophys. Res., 95, 1955-1963.

McManus, J. F., R. Francois, J.-M. Gherardi, L. D. Keigwin, and S. Brown-Leger (2004), Collapse and rapid resumption of Atlantic meridional circulation linked to deglacial climate changes, Nature, 428, 834-837.

North, G. R., R. F. Cahalan, and J. A. Coakley Jr. (1981), Energy balance climate models, Rev. Geophys., 19, 90-121.

Paillard, D. (1998), The timing of Pleistocene glaciations from a simple multiple-state climate model, Nature, 391, 378-381.

Pelletier, J. D. (2003), Coherence resonance and ice ages, J. Geophys. Res., 108(D20), 4645, doi:10.1029/2002JD003120.

Rahmstorf, S. (1995), Bifurcations of the Atlantic thermohaline circulation in response to changes in the hydrological cycle, Nature, $378,145-149$.

Raymo, M. E. (1997), The timing of major climate terminations, Paleoceanography, 12, $577-585$.

Raymo, M. E., W. F. Ruddiman, J. Backman, B. M. Clement, and D. G. Martinson (1989), Late Pleiocene variations in Northern Hemisphere ice sheets and North Atlantic deep water circulation, Paleoceanography, 4, 413-446.

Rial, J. A. (1999), Pacemaking the ice ages by frequency modulation of Earth's orbital eccentricity, Science, 285, 564-568.

Ruddiman, W., M. Raymo, D. Martinson, B. Clement, and J. Blackman (1989), Pleistocene evolution: Northern Hemisphere ice sheets and North Atlantic Ocean, Paleoceanography, 4, 353-412.

Saltzman, B. (1990), Three basic problems of paleoclimatic modeling: A personal perspective and review, Clim. Dyn., 5, 67-78.

Saltzman, B., and K. A. Maasch (1991), A firstorder global model of late Cenozoic climatic change II. Further analysis based on a simplification of $\mathrm{CO}_{2}$ dynamics, Clim. Dyn., 5, $201-$ 210 . 
Saltzman, B., and A. Sutera (1987), The MidQuaternary climate transition as the free response of a three-variable dynamical model, J. Atmos. Sci., 44, 236-241.

Sellers, W. D. (1969), A global climatic model based on the energy balance of the Earthatmosphere system, J. Appl. Meteorol., 8, $392-400$.
Stommel, H. (1961), Thermohaline convection with two stable regimes of flow, Tellus, 13, 224-230.

Tziperman, E., and H. Gildor (2003), On the mid-Pleistocene transition to $100-\mathrm{kyr}$ glacial cycles and the asymmetry between glaciation and deglaciation times, Paleoceanography, 18(1), 1001, doi:10.1029/2001PA000627.

P. D. Ditlevsen, Centre for Ice and Climate, Niels Bohr Institute, University of Copenhagen, Juliane Maries Vej 30, DK-2100 Copenhagen, Denmark. (pditlev@gfy.ku.dk) 